\title{
"It all needs to be a full jigsaw, not just bits": exploration of healthcare professionals' beliefs towards supported self-management for long-term conditions
}

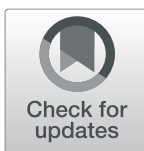

Niall Anderson ${ }^{1,2^{*}}$ (1) and Gozde Ozakinci

\begin{abstract}
Background: Long-Term Conditions are physical health issues which profoundly impact physical and psychological outcomes and have reached epidemic worldwide levels. An increasing evidence-base has developed for utilizing Supported Self-Management to ensure Health, Social Care \& Voluntary staff are knowledgeable, skilled, and experienced to enable patients to have the confidence and capability to self-manage their conditions. However, despite Health Psychology theories underpinning chronic care models demonstrating beliefs are crucially associated with intention and behaviour, staff beliefs towards Supported Self-Management have received little attention. Therefore, the study aimed to explore healthcare professionals' beliefs towards Supported Self-Management for Long-Term Conditions using the Theory of Planned Behaviour.
\end{abstract}

Methods: A mixed-methods approach was conducted within a single UK local government authority region in 2 phases: (1) Qualitative focus group of existing Supported Self-Management project staff $(N=6)$; (2) Quantitative online questionnaire of general Long-Term Conditions staff $(N=58)$.

Results: (1) Eighty two utterances over 20 theme sub-codes demonstrated beliefs that Supported Self-Management improves healthcare outcomes, but requires enhancements to patient and senior stakeholder buy-in, healthcare culture-specific tailoring, and organizational policy and resources; (2) Mean scores indicated moderate-strength beliefs that Supported Self-Management achieves positive healthcare outcomes, but weak-strength intentions to implement Supported Self-Management and beliefs it is socially normative and perceived control over implementing it. Crucially, regression analyses demonstrated intentions to implement Supported Self-Management were only associated with beliefs that important others supported it and perceived control over, or by whether it was socially encouraged.

Conclusions: Healthcare professionals demonstrated positive attitudes towards Supported Self-Management improving healthcare outcomes. However, intentions towards implementing this approach were low with staff only slightly believing important others (including patients and clinicians) supported it and that they had control over using it. Future Supported Self-Management projects should seek to enhance intention (and consequently behaviour) through targeting beliefs that important others do indeed actually support this approach and that staff have control over implementing it, as well as enhancing social encouragement.

Keywords: Long-term, Physical, Condition, Supported, Self-management, Healthcare, Health, Belief, Intention

\footnotetext{
* Correspondence: niall.anderson@ucl.ac.uk

${ }^{1}$ Public Health Department, NHS Borders, Melrose TD6 9BD, UK

${ }^{2}$ School of Medicine, University of St Andrews, St Andrews KY16 9TF, UK

(c) The Author(s). 2019 Open Access This article is distributed under the terms of the Creative Commons Attribution 4.0 International License (http://creativecommons.org/licenses/by/4.0/), which permits unrestricted use, distribution, and reproduction in any medium, provided you give appropriate credit to the original author(s) and the source, provide a link to the Creative Commons license, and indicate if changes were made. The Creative Commons Public Domain Dedication waiver (http://creativecommons.org/publicdomain/zero/1.0/) applies to the data made available in this article, unless otherwise stated.
} 


\section{Background}

\section{Long-term conditions}

Long-term Conditions (LTCs), also termed Chronic Conditions $[1,2]$, comprise complex physical health issues which require ongoing specialist support to enable patients to live with the permanent and/or disabling effects of conditions [3-5]. LTCs encompass a range of conditions which may be treated but are infrequently cured and vary in prevalence, severity, and consequences [6]. LTCs are the leading cause of premature and preventable mortality worldwide, with no country yet successfully reducing LTC levels [2, 7]. Therefore, a significant challenge is faced to target the negative impact of LTCs on life expectancy, healthy life expectancy, healthcare utilisation and expenditure, long-term sickness absence costs, disability, and the likelihood of experiencing comorbid physical health conditions [2, 8-12]. In addition to physical and medical effects, LTCs are associated with increased risk of depressive or anxiety disorders, which may have profound negative implications for self-care, symptom severity, medication adherence, health behaviours, and LTC-related relapse and survival [3, 13-19].

In addition to the medical, physical and psychological impact of individual LTCs [3], patients may also experience multi-morbid conditions where several LTCs coexist or co-morbid conditions where several LTCs stem from one Index LTC [20, 21]. 33\% UK adults and 50\% of over 60 year-olds experience at least one LTC, with two or more Multi-Morbid conditions present in 65\% 65-84 year-olds and $82 \%$ over 85 year-olds [6, 22, 23]. As a consequence, LTC patients' require 66\% NHS England expenditure, 50\% GP and 64\% outpatient appointments, and $70 \%$ acute care and inpatient bed stays [23, 24]. Furthermore, as LTCs also have profound mental health consequences [3], 9\% NHS England expenditure is required specifically for the psychological impact of LTCs [13-15, 17, 19, 25, 26]. Crucially, the challenges posed by LTCs are mirrored globally with similar epidemic levels and increases across all age groups experienced worldwide [22, 27-30]. Therefore, a movement towards a proactive, collaborative, person-focused approach supporting people to effectively manage their health is required $[6,31]$.

\section{Supported self-management}

The Chronic Care Model [32] provided an initial framework for the development of a collaborative care approach, which was subsequently supplemented by the Innovative Care for Chronic Conditions framework [5, 33] and Expanded Chronic Care Model [34]. Despite comprising different components, these models highlighted the importance of evidence-based system design, organizational and community support, and patient-professional interactions to support self-management [1]. As a consequence,
Person-Centred Care (PCC) approaches were developed which promote personalised, coordinated, enabling and respectful healthcare to support patients to have the knowledge, skills and confidence to make informed decisions about their condition(s) and treatment(s) [35]. While intuitively a common-sense approach which Health, Social Care E Voluntary (HSV) individuals may assume is already provided, there is not a universally accepted model of PCC implementation and PCC is not routinely conducted despite being central to healthcare policies in the UK and beyond [35-37].

The UK-based charity The Health Foundation's PCC review [35] outlines multiple healthcare implementation approaches, including collaborative care and support planning, experience-based co-design, person- and family-centred care, and shared decision making. However Supported Self-Management (SSM), also termed Coordinated or Integrated Care, is the most increasingly promoted and implemented PCC approach which builds upon self-care and self-management [35]. Self-care includes behaviours conducted to reduce health-impairing, and enhance health-promoting, behaviours [38], while self-management categorises taking responsibility to proactively manage condition(s) and treatment(s) [39]. SSM enhances these approaches to promote HSV knowledge, skills, experience, confidence and support to ensure patients are supported to effectively self-manage health and overcome social, personal, environmental and economic LTC challenges [6, 39].

An increasing evidence-base is emerging for SSM implementation in both generic and specific LTC settings [35]. Whole-system LTC SSM approaches include Alaska's Nuka System of Care, Germany's Proactive Chronic Care Management Program, Netherland's Buurtzorg Model, Florida's A Healthy State programme, and Hong Kong's Chronic Disease Self-Management Programme, which enhanced multiple patient, healthcare professional, and organizational outcomes [40-45]. Furthermore, SSM programmes have been a particular focus of diabetic healthcare settings $[46,47]$, including the UK's Year of Care programme [48] which developed from issues with traditional healthcare methods, patient and HSV feedback, national policies, and theoretical support for the Chronic Care Model [32]. Due to positive outcomes from the diabetes-specific programme, the LTCgeneral House of Care Model was subsequently to support collaborative care planning and processes through enabling patients, HSV staff, organizations and commissioners to promote $\operatorname{SSM}[6,49]$.

SSM programmes have resulted in improvements for patients' medical, health and preference-based treatment outcomes, HSV engagement, satisfaction and skills, patient-professional communication, activation and shared-decision making, and healthcare costs, utilization 
and adherence [35, 50-59]. However, despite the evidence-base supporting SSM, if implementation is not appropriately promoted and supported, components intended as facilitators may instead be barriers $[46,60]$. These may include patient and professional characteristics (including values, attitudes, knowledge and demographics), patient-professional interactions (including communication styles, discrepancies in understanding, and trust), LTC characteristics and treatments (including symptom presentations, multi-morbidity, and treatment availability), and organizational cultures and infrastructures (including staff availability, SSM-promotion and support) [35]. Crucially, to overcome potential barriers and facilitate the development of evidence-based SSM programmes which effectively facilitate and maintain behaviour change, an understanding of underlying psychological principles is key [24].

\section{Health, social care \& voluntary staff beliefs}

Substantial research has explored patient beliefs towards SSM and the systematic facilitators and barriers for HSV and systems to implement SSM [6, 33, 59-75]. However, a fundamental principle of the chronic care models [3234] from which SSM developed is that specific beliefs are required for a behaviour to occur [2]. Therefore, despite collaboration between patients and HSV staff being critical to whether or not SSM occurs, (to the researchers' knowledge) research has not assessed the crucial component of HSV beliefs towards SSM [24]. Health Behaviour Models seek to determine associations between health beliefs and behaviours [76]. The Theory of Planned Behaviour (TPB) is a prominent Health Behaviour Model which (like all models) is not without challenges [77-79], but as a pre-existing, validated TPB questionnaire development guide exists to facilitate the assessment of the likelihood of specific health behaviours occurring, it has provided a framework to assess HSV beliefs towards a range of behaviours including care approach, safety behaviours, hand hygiene, and identification of patients at high clinical risk [80-86].

The TPB proposes that whether one conducts a behaviour is associated with intention towards it. Intention is associated with three key direct beliefs measured through directly asking questions on these constructs, which are each influenced by two indirect beliefs which may be measured through indirectly asking about elements which may tap into direct beliefs [77, 78, 86]. Within the SSM context, Direct Attitude relates to whether SSM is perceived to have positive or negative LTC outcomes, which may be influenced by Indirect Behavioural Beliefs of perceived positive or negative consequences of SSM such as improvements to patient outcomes, combined with Indirect Outcome Evaluations of the perceived desirability of consequences. Direct
Subjective Norms relates to social pressure to conduct SSM which may be influenced by Indirect Normative Belief perceptions of what important others (such as GPs in Primary Care settings) feel about SSM, combined with Indirect Outcome Evaluation of the important of their approval. Finally Direct Perceived Behavioural Control relates to perceived efficacy to conduct SSM, which may be influenced by Indirect Control Beliefs of perceived ability to actually conduct SSM if required, combined with Indirect Influence of Control for confidence about doing so.

\section{Study objectives}

Despite SSM requiring collaborative processes between patients and HSV, (to the researchers' knowledge) an evidence-gap exists for HSV staff beliefs towards SSM. The Adapted TPB Model for Collaborative SSM (Fig. 1) demonstrates the importance of identifying and targeting both patient and HSV beliefs and intentions as involvement of both is required for SSM implementation. Therefore, the mixed-methods exploratory research project sought to use the TPB questionnaire development guide [86] to provide an initial exploration into HSV staff beliefs and intentions towards implementing SSM in LTC healthcare within a single UK local government authority region.

\section{Methods}

The mixed-methods research project was conducted in two phases based on Francis et al.'s [86] guide: (1) qualitative focus group of HSV staff from an existing SSM for LTC project (Additional file 1); (2) quantitative online questionnaire of general LTC staff (Additional file 2).

\section{Phase 1: focus group \\ Aim}

Assess beliefs towards SSM healthcare approach for LTCs in HSV staff with direct and/or indirect involvement in an existing House of Care-based project [48].

\section{Participants \& procedure}

HSV staff with direct (patient contact) and/or indirect (service level) involvement in an existing regional SSM for LTCs project were eligible. No exclusions were placed upon age, gender, race, organization, profession, type of patient contact, project involvement, time in current role, time working with LTCs, or experience of SSM. All $11 \mathrm{HSV}$ project staff members were identified and determined by the SSM Project Manager as satisfying the eligibility criteria and were invited to participate. During the focus group, the behavioural focus of 'HSV staff use of a SSM approach with LTC patients during consultations' was explored through indirect TPB-based beliefs [86-89]. Six eligible staff members volunteered to participate (2 unavailable, 3 unspecified). Participants 


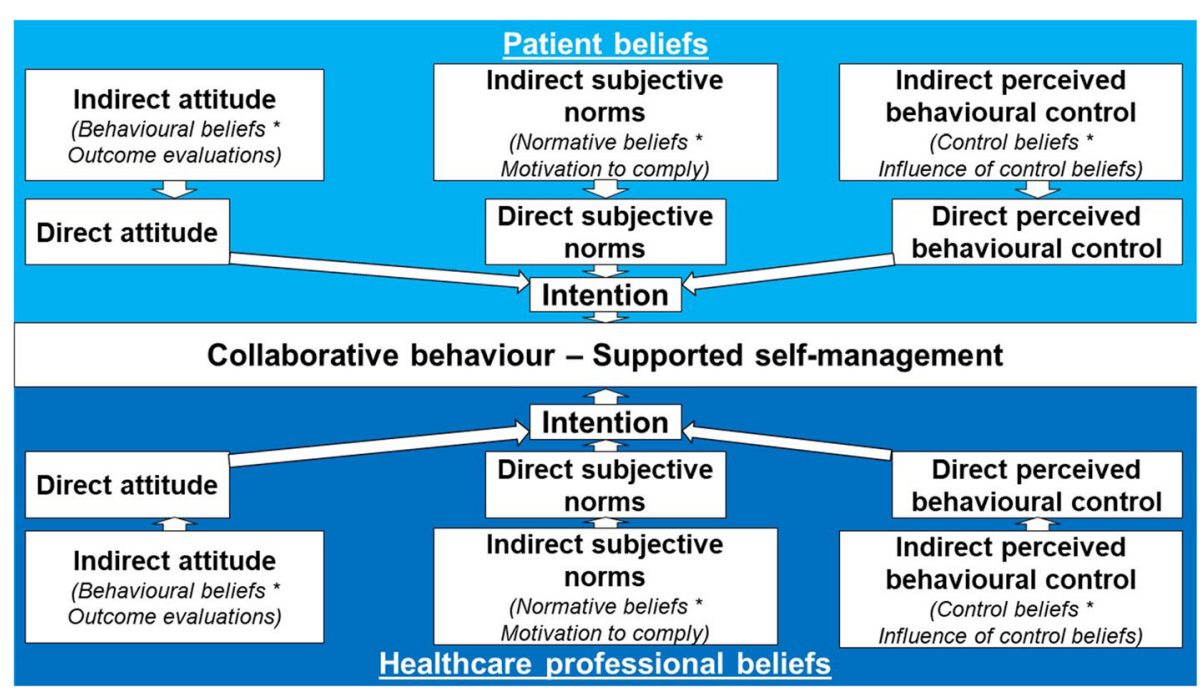

Fig. 1 Adapted Theory of Planned Behaviour for Supported Self-Management. Graphical representation (developed by NA) of how the Theory of Planned behaviour applies to collaborative behaviours which require beliefs and intentions of both patients and healthcare professionals

were representative of the overall SSM project team $(100 \%$ female/white/British $/ \geq 18$ years-old $/ \geq 10$ years working with LTC), and comprised a range of organisations (50\% NHS, 33\% local government, 17\% voluntary) and patient contact (50\% direct, $50 \%$ indirect). NA facilitated, audio recorded and transcribed [90] the $80 \mathrm{~m}: 58 \mathrm{~s}$ focus group session. A combination of inductive and deductive thematic analysis was conducted through using a TPB-based researcher-developed coding criterion and inter-rater coding to interpret transcripts in rich detail directly from participants' utterances [91, 92].

\section{Measures \& data analysis}

TPB-based belief utterances were manually coded onto the Microsoft Office Word $2007^{\mathrm{ma}}$ transcript using the researcher-developed (NA; GO) TPB coding criteria
(Table 1) $[77,78,86]$. The criteria was developed using TPB theme and sub-theme definitions, and authors' understanding of SSM literature. This required identification of potential TPB-based utterances before determining the belief and sub-belief category, and subsequently assigning a specific descriptor and code for each utterance. No minimum or maximum criterion was set for the number of words required to be coded, with potential code durations ranging from a single word to multiple sentences. As belief strength was inferred from utterance frequency (rather than duration), once a code was assigned it was not coded again until the same participant uttered a different belief or a different participant uttered any belief. Subsequently, interrater coding was conducted (between NA's and GO's codes), code frequencies calculated, and the most frequent $75 \%$ of codes used as representations of each respective belief.

Table 1 Theory of Planned Behaviour-Based Coding Criteria

\begin{tabular}{|c|c|c|c|c|}
\hline \multicolumn{2}{|l|}{ Belief Themes } & \multicolumn{3}{|c|}{ Belief Sub-Themes } \\
\hline Terminology & Definition & Terminology & Definition & Example \\
\hline \multirow[t]{2}{*}{ Behavioural } & $\begin{array}{l}\text { Belief about consequences of } \\
\text { conducting SSM. }\end{array}$ & Instrumental. & $\begin{array}{l}\text { Beliefs about what SSM will } \\
\text { achieve. }\end{array}$ & $\begin{array}{l}\text { "SSM will improve/hinder patient } \\
\text { outcomes". }\end{array}$ \\
\hline & & Experiential. & $\begin{array}{l}\text { Beliefs about how SSM feels } \\
\text { to conduct. }\end{array}$ & $\begin{array}{l}\text { "SSM feels like it will/won't be } \\
\text { positive to do". }\end{array}$ \\
\hline \multirow[t]{2}{*}{ Subjective Norm } & $\begin{array}{l}\text { Beliefs about important others } \\
\text { (e.g. patients, senior clinicians) } \\
\text { beliefs' towards SSM. }\end{array}$ & Norms. & $\begin{array}{l}\text { Beliefs about whether SSM is } \\
\text { organizationally standard } \\
\text { practice. }\end{array}$ & $\begin{array}{l}\text { "SSM is not/ promoted by the } \\
\text { organization". }\end{array}$ \\
\hline & & Pressure. & $\begin{array}{l}\text { Beliefs about how others who } \\
\text { are important to them feel } \\
\text { about SSM. }\end{array}$ & $\begin{array}{l}\text { "All/none of my colleagues think } \\
\text { SSM is positive". }\end{array}$ \\
\hline \multirow[t]{2}{*}{ Perceived Behavioural Control } & $\begin{array}{l}\text { Beliefs about control over } \\
\text { conducting SSM. }\end{array}$ & Self-Efficacy. & $\begin{array}{l}\text { Beliefs about confidence one } \\
\text { can appropriately use SSM. }\end{array}$ & $\begin{array}{l}\text { "I feel like I am not/ already able } \\
\text { to use SSM". }\end{array}$ \\
\hline & & Controllability. & $\begin{array}{l}\text { Beliefs about whether using } \\
\text { SSM is their choice. }\end{array}$ & $\begin{array}{l}\text { "SSM use is not/up to me and } \\
\text { the patient". }\end{array}$ \\
\hline
\end{tabular}




\section{Phase 2: online questionnaire of LTC staff}

Aim

Assess beliefs towards adopting SSM healthcare approach for LTCs in general HSV staff.

\section{Participants \& procedure}

Researchers developed a questionnaire (Additional file 2) based on Phase 1 TPB-based beliefs, comprising 51items across 4 overarching variables: Demographics, Intention, Beliefs and Optional Feedback. This was published online using SurveyMonkey Gold@, before being piloted and deemed suitable by 5 HSV staff with no formal experience of a SSM project. Staff were approximately representative of regional LTC healthcare $(60 \%$ female; $100 \%$ white/British/ $\geq 18$ years-old), comprised a range of organizations ( $60 \%$ NHS, $40 \%$ local government) and patient contact (60\% direct, $40 \%$ indirect), and all had over 10 years' experience of LTC.

HSV staff working with LTCs in any capacity were eligible to participate, with no exclusions placed upon age, gender, race, organization, profession, type of patient contact, project involvement, time in current role, time working with LTCs, or experience of SSM. Two complimentary recruitment methods were utilized. First, a senior healthcare line manager directly e-mailed 35 senior, regional HSV line managers to request the further dissemination of an e-mail invitation to participate. Second, regional HSV communications departments disseminated the questionnaire to wider HSV staff internally. As participants were able to omit responses to specific questions, the proportion of the questionnaire completed varied. However, in order to conduct regression analyses responses were required for all subvariables. Therefore, participants were classed as Completers if they omitted a maximum of 1 response to each sub-variable prior to imputation. As a consequence, 115 participants (Table 2) were separated into three completion level groups (Demographic-Only: $n=20$; Demographic $\mathcal{E}$ Intention: $n=37$; Completers: $n=58$ ) and demographic differences between completion-level groups assessed prior to Completer group regressions. Chi-square tests demonstrated no demographic differences based on survey completion-level (DemographicsOnly; Demographics \& Intention; Completers) for age, gender, organization, service, and time within current role $(p>.05)$. However, completers demonstrated significantly greater LTC patient contact $\left(\mathrm{Chi}^{2}(8)=25.196\right.$, $p=.001)$, and experience both of LTC $\left(\mathrm{Chi}^{2}(8)=16.946\right.$, $p=.031)$ and SSM $\left(\mathrm{Chi}^{2}(10)=25.812, \mathrm{p}=.001\right)$.

Data were collated and analysed using IBM SPSS Statistics $23^{\text {тм}}$. Data screening, scoring, imputation, internal consistency and variable computation procedures were conducted in accordance with Francis et al.'s [86] procedures. First, individual item responses were scored using either a unipolar scale of 1-7 for concepts which unidirectional measurement was appropriate (e.g. probability), or a bipolar scale of \pm 3 for concepts which bidirectional measurement was appropriate (e.g. evaluation). Lower scores (e.g. 1 or -3) reflected 'Strong Negative Beliefs' towards SSM, mid-range scores (e.g. 4 or 0) reflected 'Neutral' beliefs with no negative or positive appraisal of SSM, and higher scores (e.g. 7 or +3 ) reflected 'Strong Positive Beliefs' towards SSM. Second, where $\leq 5 \%$ data is missing effects are deemed negligible and no single imputation approach is most effective [93-98]. Therefore, item-average data imputation was conducted for the missing $0.37 \%$ of intention or belief responses. Third, internal consistency was analysed and specific items removed from sub-variables to maximise internal consistency. Fourth, composite variable scores were calculated and interpreted in accordance with Francis et al.'s procedure [86]. For direct beliefs, SPSS was used to 'compute' composite scores for direct measures. For indirect beliefs, each indirect belief sub-domain (e.g. behavioural belief question 1) was multiplied by its respective indirect belief sub-domain (e.g. outcome evaluation question 1), before all weighted belief scores were summed to create a composite belief score (e.g. indirect attitude). Finally, regression analyses were conducted on the 'Completer' sample of participants who satisfied the aforementioned questionnaire completion criteria in order to determine whether beliefs significantly associated with intention to implement SSM.

\section{Measures \& data analysis}

The Completer sample $(n=58)$ data was analysed using IBM SPSS Statistics $23^{\mathrm{Tm}}$ with two multiple regressions to determine whether direct and indirect beliefs (independently) associated with intention to implement SSM, and three (independent) linear regressions to determine whether indirect beliefs associated with their respective direct belief (Additional file 3).

\section{Results}

\section{Phase 1: focus group}

Following an initial 94\% belief utterance and 100\% code agreement between researchers, 82 utterances across 20 belief codes were agreed upon and the most representative beliefs determined based on utterance frequency (Table 3). Beliefs indicated SSM:

1. Improves holistic healthcare provision, communication channels and demands, but requires staff to be supported through simplified organizational pathways to be effective.

2. Effectively applied in other healthcare settings but patients may not always want or understand SSM. Furthermore, to be effective SSM must be tailored 
Table 2 Participant Demographic Information

\begin{tabular}{|c|c|c|c|}
\hline \multicolumn{2}{|l|}{ Demographic Information } & \multicolumn{2}{|l|}{ Participants } \\
\hline Category & Response Options & Overall $(n=115)$ & Completers $(n=58)$ \\
\hline \multirow[t]{4}{*}{ Age } & $18-24 y$ & $3 \%$ & $4 \%$ \\
\hline & $25-39 y$ & $15 \%$ & $10 \%$ \\
\hline & $40-60 y$ & $76 \%$ & $83 \%$ \\
\hline & $>60 y$ & $6 \%$ & $3 \%$ \\
\hline \multirow[t]{3}{*}{ Gender } & Female & $80 \%$ & $78 \%$ \\
\hline & Male & $16 \%$ & $17 \%$ \\
\hline & No response & $4 \%$ & $5 \%$ \\
\hline \multirow[t]{6}{*}{ Organisation } & $\mathrm{NHS}$ & $59 \%$ & $60 \%$ \\
\hline & Council & $28 \%$ & $24 \%$ \\
\hline & Voluntary & $8 \%$ & $9 \%$ \\
\hline & Multiple & $2 \%$ & $4 \%$ \\
\hline & Other & $1 \%$ & $0 \%$ \\
\hline & No response & $2 \%$ & $3 \%$ \\
\hline \multirow[t]{6}{*}{ Service } & Primary care & $21 \%$ & $26 \%$ \\
\hline & Secondary care & $17 \%$ & $19 \%$ \\
\hline & Community & $42 \%$ & $34 \%$ \\
\hline & Multiple & $13 \%$ & $9 \%$ \\
\hline & Other & $5 \%$ & $12 \%$ \\
\hline & No response & $2 \%$ & $0 \%$ \\
\hline \multirow[t]{5}{*}{ LTC patient contact } & Direct & $38 \%$ & $38 \%$ \\
\hline & Indirect & $9 \%$ & $3 \%$ \\
\hline & Direct \& indirect & $49 \%$ & $57 \%$ \\
\hline & None & $3 \%$ & $0 \%$ \\
\hline & No response & $1 \%$ & $2 \%$ \\
\hline \multirow[t]{5}{*}{ Time in role } & $<1 y$ & $9 \%$ & $14 \%$ \\
\hline & $2-5 y$ & $23 \%$ & $22 \%$ \\
\hline & $6-9 y$ & $19 \%$ & $17 \%$ \\
\hline & $>10 y$ & $46 \%$ & $47 \%$ \\
\hline & No response & $3 \%$ & $0 \%$ \\
\hline \multirow[t]{5}{*}{ Time working with LTC } & $<1 y$ & $5 \%$ & $5 \%$ \\
\hline & $2-5 y$ & $12 \%$ & $10 \%$ \\
\hline & $6-9 y$ & $13 \%$ & $12 \%$ \\
\hline & $>10 y$ & $64 \%$ & $71 \%$ \\
\hline & No response & $6 \%$ & $2 \%$ \\
\hline \multirow[t]{5}{*}{ SSM involved in current role } & Never & $22 \%$ & $12 \%$ \\
\hline & Sometimes & $39 \%$ & $31 \%$ \\
\hline & Often & $25 \%$ & $36 \%$ \\
\hline & Always & $12 \%$ & $19 \%$ \\
\hline & No response & $2 \%$ & $2 \%$ \\
\hline
\end{tabular}


Table 3 Theory of Planned Behaviour-Based Beliefs - Focus Group Sample

\begin{tabular}{|c|c|c|c|c|c|c|}
\hline \multicolumn{4}{|l|}{ Belief } & \multicolumn{2}{|c|}{$\begin{array}{l}\text { Utterance } \\
\text { Frequency }\end{array}$} & \multirow{2}{*}{$\begin{array}{l}\text { Phase 2? } \\
\text { (Yes/No) }\end{array}$} \\
\hline Category & Sub-Belief & Code & Description & No. & Rank & \\
\hline \multirow[t]{7}{*}{ Behavioural } & \multirow[t]{6}{*}{ Instrumental } & BB1 & SSM requires support from HSV staff in order to be effective. & 1 & 7 & NO \\
\hline & & BB2 & $\begin{array}{l}\text { Additional/simplified organizational pathways are required in order } \\
\text { for SSM to achieve positive outcomes. }\end{array}$ & 5 & 2 & YES \\
\hline & & BB3 & SSM improves communication channels. & 4 & 3 & YES \\
\hline & & BB4 & SSM improves holistic healthcare provision. & 7 & 1 & YES \\
\hline & & BB5 & SSM improves patient outcomes. & 2 & 5 & NO \\
\hline & & BB7 & SSM reduces healthcare time demands. & 2 & 5 & YES \\
\hline & Experiential & BB6 & SSM is not possible if staff are not supported and facilitated to use it. & 3 & 4 & YES \\
\hline \multirow[t]{7}{*}{ Subjective Norm } & \multirow[t]{6}{*}{ Norms } & NB1 & SSM is effectively being applied in other areas/regions. & 2 & 3 & YES \\
\hline & & NB2 & SSM is promoted by HSV policy and documentation. & 1 & 6 & NO \\
\hline & & NB3 & $\begin{array}{l}\text { Patients may not always understand, or want staff to implement, SSM } \\
\text { healthcare. }\end{array}$ & 7 & 1 & YES \\
\hline & & NB4 & $\begin{array}{l}\text { Widespread use of SSM would be required in order for it be effectively } \\
\text { adopted. }\end{array}$ & 1 & 6 & NO \\
\hline & & NB5 & $\begin{array}{l}\text { SSM must factor in cultural/ local norms of different HSV settings to } \\
\text { be effective. }\end{array}$ & 2 & 3 & YES \\
\hline & & NB7 & Patients want to be involved and understand their medication regimens. & 2 & 3 & YES \\
\hline & Pressure & NB6 & Without GP buy-in the implementation of a SSM approach is not possible. & 5 & 2 & YES \\
\hline \multirow{6}{*}{$\begin{array}{l}\text { Perceived Behavioural } \\
\text { Control }\end{array}$} & \multirow[t]{2}{*}{ Self-Efficacy } & CB1 & SSM requires effective co-produced healthcare. & 1 & 5 & NO \\
\hline & & CB6 & $\begin{array}{l}\text { SSM training must be tailored to staff knowledge, skills, experience and } \\
\text { needs. }\end{array}$ & 8 & 3 & YES \\
\hline & \multirow[t]{4}{*}{ Controllability } & CB2 & SSM is limited by HSV policy and capacity. & 9 & 2 & YES \\
\hline & & CB3 & Resource investments are required to increase staff SSM control. & 13 & 1 & YES \\
\hline & & CB4 & SSM requires increased staff engagement to enhance control. & 6 & 4 & YES \\
\hline & & CB5 & $\begin{array}{l}\text { IT/communication sharing improvements are required to enhance staff } \\
\text { control. }\end{array}$ & 1 & 5 & NO \\
\hline
\end{tabular}

to specific healthcare settings and receive senior clinician buy-in (especially GPs in Primary Care settings).

3. Implementation limited by healthcare policy, staff engagement, and in particular resource investments. Healthcare culture-specific training which is tailored specifically to staff's knowledge, skills and experience is required.

\section{Phase 2: online questionnaire of LTC staff}

The 58 'Completers' (Table 2) demonstrated moderatestrength positive attitude beliefs, and weak-strength positive intention, subjective norm, and perceived behavioural control beliefs (Table 4). Figure 2 represents the significance and effect sizes analyses outlined by the TPB questionnaire development guide [86]. This includes two independent multiple regression analyses conducted to determine the associations of overall direct and indirect beliefs with intention, and three independent linear regression analyses conducted to determine the associations of indirect beliefs with their respective direct belief.
The 'Combined Direct Beliefs' multiple regression model significantly explained $37.2 \%$ of variance in 'Intention' to conduct SSM with LTCs $(\mathrm{F}(3,53)=$ 10.482, $p<.001, \mathrm{R}^{2}=.372, \mathrm{R}_{\text {Adjusted }}^{2}=.337$ ), with intention significantly explained by the individual predictor variables of 'Direct Subjective Norms' $(\beta=.408, \mathrm{t}$ $(53)=3.122, p=.003)$ and 'Direct Perceived Behavioural Control' $(\beta=.319, \mathrm{t}(53)=2.585, p=.013)$, but not 'Direct Attitude' $(\beta=-.003, \mathrm{t}(53)=-.024, p=.981)$. 'Combined Indirect Beliefs' significantly explained $24.7 \%$ of variance in 'Intention' (F $(3,54)=5.893, p=.001$, $\mathrm{R}^{2}=.247, \mathrm{R}^{2}$ Adjusted $=.205$ ), with 'Intention' significantly associated with 'Indirect Subjective Norms' $(\beta=.438, \mathrm{t}$ $(54)=3.460, \quad \mathrm{p}=.001)$, but not 'Indirect Attitude' $(\beta=.156, \mathrm{t}(54)=.951, p=.346)$ or 'Indirect Perceived Behavioural Control' $(\beta=-.034, \mathrm{t}(54)=-.213, p=.832)$. Linear regression models demonstrated that 'Indirect Attitude' significantly explained $10.6 \%$ of variance in 'Direct Attitude' (F $(1,55)=6.516, \mathrm{p}=.013, \mathrm{R}^{2}=.106, \mathrm{R}^{2}$ Adjusted $=.090$ ), and 'Indirect Subjective Norms' significantly explained $40.6 \%$ of variance in 'Direct Subjective Norms' 
Table 4 'Completer' Sample Theory of Planned Behaviour-Based Descriptive Statistics

\begin{tabular}{|c|c|c|c|c|c|c|c|}
\hline \multirow[t]{2}{*}{ Component } & \multirow{2}{*}{$\begin{array}{l}\text { Intention } \\
\text { Generalised } \\
\text { Mean }\end{array}$} & \multicolumn{2}{|l|}{ Attitude } & \multicolumn{2}{|l|}{ Subjective Norm } & \multicolumn{2}{|c|}{ Perceived Behavioural Control } \\
\hline & & Direct Mean & Indirect Sum & Direct Mean & Indirect Sum & Direct Mean & Indirect Sum \\
\hline$n$ & 58. & 58. & 58. & 58. & 58. & 58. & 58. \\
\hline Mean & 5.155 & 5.868. & 49.560. & 4.922. & 19.569. & 4.922. & 21.839. \\
\hline SD & 1.832. & 1.292 & 33.055. & 1.363. & 21.186. & 1.217 & 129.00 \\
\hline Standardized Mean (1-7) & 5.155 & 5.868 & 5.565 & 4.922 & 4.152 & 4.922 & 4.401 \\
\hline Interpretation & $\begin{array}{l}\text { Weak-Strength } \\
\text { Positive Belief. }\end{array}$ & $\begin{array}{l}\text { Moderate-Strength } \\
\text { Positive Belief. }\end{array}$ & $\begin{array}{l}\text { Moderate-Strength } \\
\text { Positive Belief. }\end{array}$ & $\begin{array}{l}\text { Weak-Strength } \\
\text { Positive Belief. }\end{array}$ & $\begin{array}{l}\text { Weak-Strength } \\
\text { Positive Belief. }\end{array}$ & $\begin{array}{l}\text { Weak-Strength } \\
\text { Positive Belief. }\end{array}$ & $\begin{array}{l}\text { Weak-Strength } \\
\text { Positive Belief. }\end{array}$ \\
\hline
\end{tabular}

Interpretation of Standardized Mean - Favourability, Less than $4=$ Negative; 4 = Neutral; Greater than $4=$ Positive

Interpretation of Standardized Mean - Strength, 3-5= Weak; $2-3$ or 5-6 Moderate; $1-2$ or $6-7=$ Strong

$\left(\mathrm{F}(1,56)=38.348, p<.001, \mathrm{R}^{2}=.406, \mathrm{R}_{\text {Adjusted }}^{2} .396\right)$. However, 'Indirect Perceived Behavioural Control' nonsignificantly explained $2.9 \%$ of variance in 'Direct Perceived Behavioural Control' $(\mathrm{F}(1,55)=1.652, p=.204$, $\mathrm{R}^{2}=.029, \mathrm{R}_{\text {Adjusted }}^{2} .012$ ).

\section{Discussion}

Research previously explored the relative strengths and challenges of SSM [35], and the patient, communicative, and organizational components associated with enhance outcomes [2, 6, 33, 40-45, 60-73]. However, despite chronic conditions models [5, 32, 34] which form the foundation of SSM placing emphasis on beliefs being fundamental to behaviour, and health psychology theory demonstrating that intention is associated with actual behaviour [77, 78], staff intentions and beliefs towards SSM have received little research attention. As the Adapted TPB for SSM Model (Fig. 1) demonstrates, this means that a significant part of the picture of what is required in order for SSM to be effectively implemented is currently unknown. Therefore, the mixed-methods research project sought to provide an initial exploration into a SSM research gap by determining the likelihood of SSM being implemented in a rural region where SSM is promoted by assessing HSV beliefs towards SSM and which beliefs are associated with intention towards implementing this approach.

The focus group demonstrated staff from an existing SSM project believe that SSM has positive implications for healthcare outcomes, but is unlikely to be effective

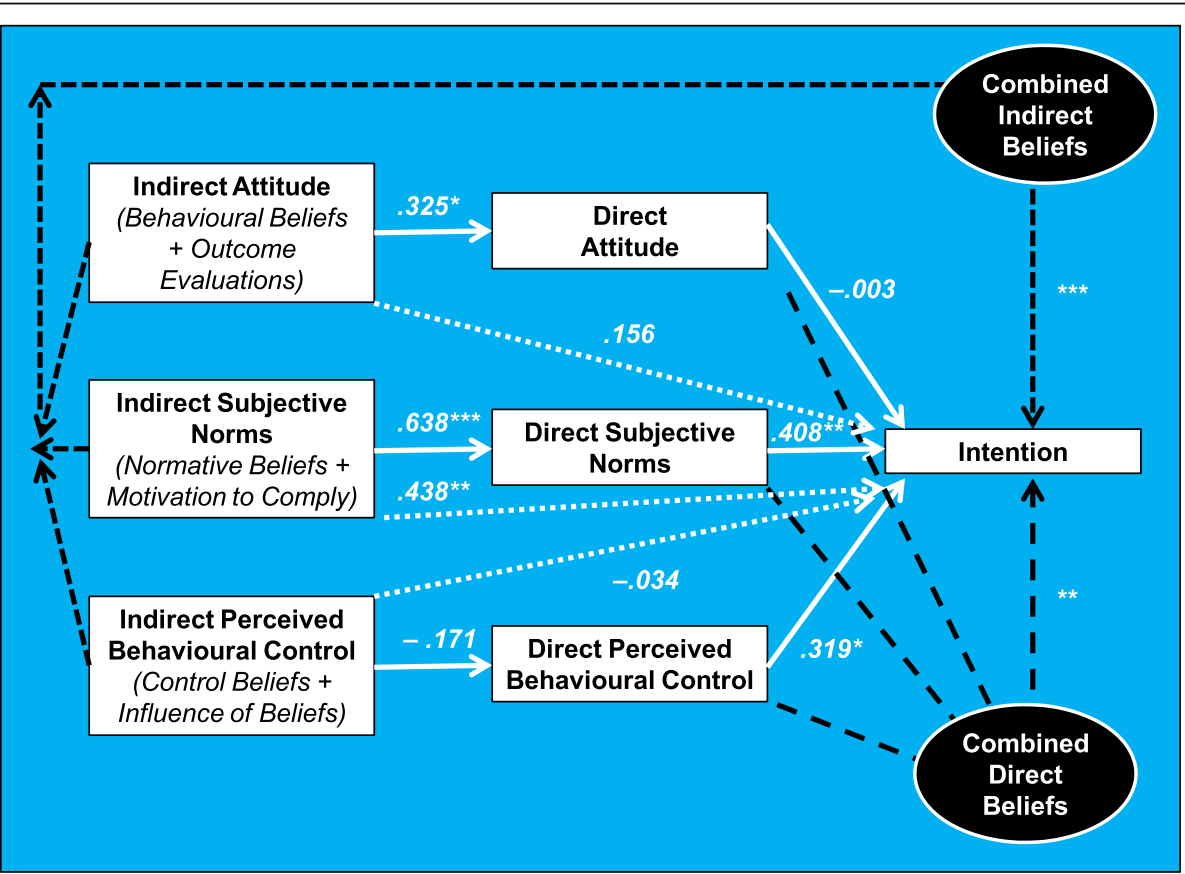

Significance Level: ${ }^{*} p<.05 ;{ }^{* *} p<.01 ;{ }^{* * *} p<.001$

Fig. 2 Regression Analyses Summary. Graphical representation (developed by NA) of how the regression analyses conducted on the Completer sample relate to the components of the Theory of Planned Behaviour 
unless enhancements are made to organizational policy, resources, senior clinician buy-in, and healthcare culture-specific training. The online questionnaire also demonstrated that general LTC staff believe that SSM has positive implications for healthcare outcomes. However, staff were unlikely to implement SSM due beliefs that they had limited ability or control over attempting this approach, and that there was limited social encouragement towards it. Crucially, staff attitudes towards whether SSM was beneficial or achieved positive outcomes did not explain intention towards implementing it. However, if staff did not feel important others (such as GPs in primary care settings) supported SSM and that they had control over using it, or they did not receive positive social encouragement towards it, staff were unlikely to intend to implement SSM.

As aforementioned, attitudes towards whether SSM achieves positive outcomes or whether they were in favour of using it did not explain intentions towards it, which may have two potential implications which require further research. First, if attitudes towards SSM do not explain intention towards implementing it then healthcare initiatives which focus on enhancing attitudes are unlikely to be effective, and focusing on alternative beliefs may be a more appropriate use of time and resources. Alternatively, as both focus group and online questionnaire samples demonstrated positive attitudes towards SSM, this possibly reflects that focusing on enhancing attitudes for those with pre-existing positive attitudes is unlikely to result in a significant enhancement. Therefore, future research should sample those with a greater range of views about whether SSM (if effectively conducted) has positive outcomes, in order to guide the degree to which attitudes influence SSM implementation.

While attitude did not associate with intention, intention towards SSM was significantly explained by beliefs for whether important others supported SSM implementation and perceived control over whether or not it should be implemented. This has two key implications for current issues which could potentially be used as opportunities to target and enhance intention towards SSM via social norm and control beliefs. First, participants had less intention to implement SSM if they perceived patients did not understand or want this approach, senior clinicians did not support it, or that SSM had not been tailored to the specific healthcare culture. However, participants believed that SSM had been effectively applied in other healthcare context. As research indicates that patients generally support SSM [2, 32], including patients in SSM initiatives to provide their direct experiences and perspectives may potentially enhance staff intentions towards SSM. Additionally, as staff perceived that SSM had been effectively implemented in other healthcare settings, including staff from other successful projects to prove information and assist the development of tailored programmes may also enhance intention. Second, participants demonstrated lower intentions towards SSM if they perceived they had low control or ability to implement SSM, which stemmed from beliefs that organizational policy and culturespecific training enhancements were required to increase engagement. Similarly, learning from previous successful programmes may also have a positive impact upon intention towards SSM. Hence, social and control beliefs significantly associated with intention towards SSM, and provide an opportunity to enhance SSM implementation through including patients and stakeholders from previous successful SSM programmes to enhance components believed to be confounds for successful implementation.

Based on TPB principles it would be expected that indirect beliefs would only associate with intention via direct beliefs. However, contrary to these principles, indirect social beliefs significantly directly associated with intention, indicating that if one perceives sufficient social encouragement towards SSM then they are significantly more likely to intend to implement SSM (independent of all other beliefs). This provides promise for SSM healthcare research and practice as it potentially indicates that, if appropriate consideration is given to promoting and supporting staff to feel encouraged to implement SSM, staff are significantly more likely to intend to do so. However, this raises an ethical issue as increasing social pressure in this context requires encouraging staff in a positive, supportive manner to promote SSM as social normative and supported by organizations, rather than negatively pressuring staff that negative consequences will occur if they are unable to implement SSM. Hence, social encouragement towards conducting SSM significantly associated with intention towards implementing this approach, and future research should seek to determine how this may be optimally achieved.

To the researchers' knowledge no previous research has assessed HSV staff beliefs towards SSM. As intention is associated with actual behaviour [77, 78], this exploratory study sought to provide an initial investigation into a key element of SSM and determine beliefs that may be used for increasing intention towards implementing SSM. However, the study only provided a starting point for psychological research into staff beliefs towards SSM for LTCs, with key considerations required. First, the study sought to determine beliefs that associate with intention to conduct SSM, which in turn is believed to associate with actual behaviour. Furthermore, as previous studies have assessed patient beliefs towards SSM, the study only explored staff beliefs towards implementing SSM. However, future studies should incorporate both patient and 
HSV beliefs in addition to measures of actual behavioural changes pre- and post-implementation of SSM programmes to determine actual behaviour in accordance with the Adapted TPB for SSM Model (Fig. 1). Second, while the TPB provided a useful framework for determining intention towards implementing SSM, direct and indirect beliefs only explained 37.2 and $24.7 \%$ of variance in intention respectively. Therefore, future research should determine the extent to which beliefs included in alternative health psychology models [76], and external constructs relating to patient and organisational barriers and facilitators, influence intention. Hence, this study provided a useful exploration into staff beliefs towards implementing SSM with LTCs patients and highlighted areas of potential focus for enhancing intentions towards implementing SSM, but future research is required to build upon these exploratory findings.

\section{Strengths \& limitations}

To the researchers' knowledge, the exploratory project was the first to use psychological principles that underlie chronic care models to assess staff beliefs towards SSM $[2,5,32,34,62,64-72]$. The TPB was selected due to having a strong pre-existing research base for assessing beliefs towards various health behaviours combined with having a pre-existing, validated questionnaire development guide [80, 81, 86, 99, 100]. However, within health psychology there is ongoing debate on whether issues with parsimony, predictive validity and utility mean that the TPB should be 'retired' in favour of alternative models, or whether aforementioned issues stem from misunderstanding TPB components and research [7779]. As highlighted by Sniehotta et al.'s [79] comprehensive critique of TPB, while Intention and Perceived Behavioural Control are relatively consistent predictors of behaviour and interventions targeting intention are likely to result in behaviour change, a significant confound of the TPB is the 'Intention-Behaviour Gap' which categorises discrepancies between these constructs which cannot be accounted for by TPB components alone and is a key area of ongoing research. Therefore, the approach adopted provided an established, evidence-based psychological framework for exploring and assessing a SSM research gap. However, future research should consider the efficacy of trialling and contrasting alternative models and frameworks [76] to determine the psychological constructs most relevant to SSM.

The TPB questionnaire development guide ensured beliefs were representative of regional healthcare cultures across a range of organisations and roles, including those with no formal SSM experience. While mixedmethods studies may be more complex, time, and resource intensive than quantitative or qualitative methods individually, they may offset the weaknesses of each approach through combining inductive and deductive reasoning to assess both observational and statistical information, and reduce potential researcher-biases [101103]. However, construct validity issues were present which may reflect questionnaire development guide and/ or measure construction confounds. First, to promote coherence of focus group discussions, TPB-based beliefs were indirectly explored through questioning the facilitators and barrier of an existing SSM project. However, direct questioning of specific TPB-based beliefs may potentially generate a more focussed exploration of beliefs. Second, pilot feedback indicated that the questionnaire was representative of intended beliefs, but may benefit from containing less questions. As TPB development guide requirements meant this was not possible and only $50.4 \%$ participants completed the questionnaire, a more flexible approach to questionnaire development may be beneficial. Finally, specific variables were removed from composite scores to improve internal consistency. However, as one belief was ultimately only represented by two sub-variables, this may indicate that specific questions may not have been optimally representative of intended beliefs and/or were sub-optimally constructed. Hence, the TPB questionnaire development guide provided a useful framework belief but future research should build upon construct confounds to improve validity.

The small, single rural geographical region where healthcare is integrated and SSM is promoted was a relevant research setting, but may have raised generalisability and recruitment confounds. First, while different SSM programmes have achieved positive outcomes across cultures [40-45], rural settings experience the dual challenge of increasingly elderly populations with higher LTC levels and recruitment difficulties compared to urban settings [104]. This may influence staff beliefs and consequently intervention implementation requirements. Second, to enhance recruitment a combination of intranet and senior HSV stakeholder e-mail invitation approaches were used. However, as existing IT mechanisms cannot accurately determine who disseminated or accessed the questionnaire, recruitment may have been confounded by senior stakeholders' personal beliefs towards SSM reducing dissemination and/or potential participants' perceptions of lacking time or capacity. Therefore, it is uncertain whether greater questionnaire completion levels by community than hospital staff was due to a lack of awareness, engagement or time for either the questionnaire or SSM in general. Hence, future research may benefit from conducting a cross-regional approach, early targeting and engagement of patients and senior stakeholders, and supplementary recruitment methods to maximise dissemination and completion. For example, involving Patient \& Public Involvement or 
HSV management groups in the design and recruitment processes, and visiting staff on hospital wards to explain the project and provide hard copies of questionnaires.

Despite attempts to promote participation from a range of SSM and LTCs experience, two key implications are present from the Completer sample being smaller than desired and having high levels of patient contact and both SSM and LTC experience. First, experience levels appear to have influenced perceived confidence, motivation and/or importance of participating but, as the first exploration of staff beliefs towards implementing SSM, uncertainty remains for the generalisability of findings and whether greater experience of LTCs and/or SSM enhances or inhibits beliefs towards implementing SSM. Second, only $50.4 \%$ of 115 participants met the questionnaire completion criterion, which has implications for ethical requirements and analyses power. As ethical requirements ensured participants could omit responses to questions but in order to also satisfy regression requirements a strict completion criterion was set. This raised a significant confound that the Completer sample of 58 participants was adequately powered for linear but not multiple regressions, and consequently results must be interpreted as an exploratory foundation for future research to replicate and develop upon, rather than definitive findings. Therefore, while the experienced sample provided valuable information, future replications comprising a larger sample with a greater range of experience is required to increase generalisability and reliability.

Finally, a key strength was the study rationale. Despite comprising different components, chronic care (and consequently SSM) models are fundamentally based on psychological theory [5, 6, 32, 34, 49]. However, despite all models proposing that SSM encompasses a combination of patient, professional, communication, and organizational factors, SSM implementation has typically occurred in the absence of research to determine staff beliefs towards SSM. Therefore, despite active attempts to enhance SSM implementation and outcomes, through neglecting HSV beliefs a significant component required to effectively implement SSM is unknown. Hence, while future research is required to build upon aforementioned strengths and improve limitations, the study highlights the importance of not only assessing and improving patient and organizational outcomes, but also HSV SSM beliefs and subsequently behaviours.

\section{Conclusions}

Despite SSM requiring collaborative behaviours between patients and clinicians, and health psychology theory which underpins SSM proposing beliefs are critical to behaviours, little research has assessed healthcare professionals' beliefs towards SSM. The findings of the exploratory study indicate that staff experienced in both SSM and LTC demonstrate moderate-strength beliefs that SSM improves holistic healthcare outcomes. However, in spite of positive attitudes, staff demonstrated weak-strength intentions to implement SSM, and weakstrength beliefs that SSM is socially encouraged, promoted by important others, and that they had the perceived control and ability to implement it. Additionally, intentions towards conducting SSM was only associated with beliefs about whether they had the choice and ability to actually implement it, or whether SSM was socially encouraged. Therefore, while staff believe that SSM improves outcomes, future healthcare research and provision is required which targets and enhances the aforementioned beliefs (as well as organizational and external factors influencing these beliefs) to increase SSM implementation.

\section{Additional files}

Additional file 1: Evaluation of perceptions of shared-management provision for those with long-term conditions in NHS borders. (PDF 435 $\mathrm{kb})$

Additional file 2: Supported self-management for people living with long-term conditions. (PDF 899 kb)

Additional file 3: Statistical analyses. (DOCX $12 \mathrm{~kb}$ )

\section{Abbreviations}

HBM: Health belief model; HSV: Health, Social Care and Voluntary; LTC: Longterm conditions; PCC: Person-Centred Care; SSM: Supported self-

management; TPB: Theory of planned behaviour

\section{Acknowledgements}

NA would like to thank Julie Murray, Dr. Allyson McCollam, and Joy Dawson at NHS Borders, and Dr. Hannah Dale and Dr. Lloyd Wallace at NHS Education for Scotland, for their support and advice throughout.

\section{Authors' contributions}

NA was involved in all processes, including: research, design, development, data collection, coding and analysis, and report formulation. GO contributed to research design and inter-rater coding and analysis, and provided supervision, advice and feedback throughout the research project.

\section{Authors' information}

NA is a Chartered Health Psychologist and is employed as a Senior Research Associate by the University College London Centre for Behaviour Change, conducting research for the National Institute for Health Research Health Protection Research Unit and Public Health England. The research project was conducted during NA's employment as a Trainee Health Psychologist by NHS Borders.

$\mathrm{GO}$ is a Chartered Health Psychologist and is a Senior Lecturer in Health Psychology within the University of St Andrews School of Medicine Division of Population and Behavioural Health Sciences.

\section{Funding}

The authors declare that they did not receive any financial support for the present study.

\section{Availability of data and materials}

The datasets used and/or analysed during the current study are available from the corresponding author on reasonable request. 


\section{Ethics approval and consent to participate}

Ethical approval for the Service Evaluation was obtained from NHS Borders Governance (SE02/16: 07/10/2016) and the University of St Andrews Teaching \& Research Ethics Committee (MD12444: 19/10/2016). Focus group participants were required to read a paper information sheet and voluntarily sign a written consent form. Online questionnaire participants were required to read an online ethics information section prior to conducting the questionnaire, which outlined that by voluntarily completing the questionnaire (or specific sections) this would be taken as providing consent.

\section{Consent for publication}

Not applicable.

\section{Competing interests}

The authors declare that they have no competing interests.

Received: 10 December 2018 Accepted: 12 June 2019 Published online: 24 June 2019

\section{References}

1. Epping-Jordan JE, Pruitt SD, Bengoa R, Wagner EH. Improving the quality of healthcare for chronic conditions. Qual Saf Health Care. 2004;14(4):299-305.

2. Nolte E, McKee M. Caring for people with chronic conditions: a health system perspective. 1st ed. London: McGraw-Hill Education; 2008

3. Anderson N, Ozakinci G. Effectiveness of psychological interventions to improve quality of life in people with long-term conditions: rapid systematic review of randomised controlled trials. BMC Psychol. 2018;6(1):11.

4. Timmreck TC. Dictionary of health services management. 1st ed. National Health Pub; 1987

5. World Health Organization. Innovative care for chronic conditions: building blocks for actions: global report. World Health Organisation. 2002.

6. Coulter A, Roberts S, Dixon A. Delivering better services for people with long-term conditions: building the house of care. 1st ed. London: The King's Fund; 2013

7. World Health Organization. Global health risks: mortality and burden of disease attributable to selected major risks. World Health Organization; 2009.

8. Goetzel RZ, Long SR, Ozminkowsi RJ, Hawkins K, Wang S, Lynch W. Health, absence, disability, and presenteeism cost estimates of certain physical and mental health conditions affecting US employers. J Occup Environ Med. 2004;46(4):398-412.

9. Henderson M, Glozier N, Elliott KH. Long term sickness absence: is caused by common conditions and needs managing. BMJ. 2005;802(7495):330.

10. Hoffman C, Rice D, Sung HY. Persons with chronic conditions: their prevalence and costs. Jama. 1996;276(18):1473-9.

11. Wilson T, Buck D, Ham C. Rising to the challenge: will the NHS support people with long term conditions? BMJ. 2005;330(7492):657.

12. Yach D, Hawkes C, Gould CL, Hofman KJ. The global burden of chronic diseases: overcoming impediments to prevention and control. Jama. 2004; 291(21):2616-22

13. Cohen S, Herbert TB. Health psychology: psychological factors and physical disease from the perspective of human psychoneuroimmunology. Annu Rev Psychol. 1996:47(1):113-42.

14. Davies SJ, Jackson PR, Potokar J, Nutt DJ. Treatment of anxiety and depressive disorders in patients with cardiovascular disease. BMJ. 2004; 328(7445):939.

15. Frasure-Smith $N$, Lesperance $F$, Juneau M, Talajic M, Bourassa MG. Gender, depression, and one-year prognosis after myocardial infarction. Psychosom Med. 1999;61(1):26-37.

16. Goodwin N, Curry N, Naylor C, Ross S, Duldig W. Managing people with long-term conditions. 1st ed. London: King's Fund; 2010.

17. Moussavi S, Chatterji S, Verdes E, Tandon A, Patel V, Ustun B. Depression, chronic diseases, and decrements in health: results from world health surveys. Lancet. 2007;370(9590):851-8.

18. Prince M, Patel V, Saxena S, Maj M, Maselko J, Phillips MR, Rahman A. No health without mental health. Lancet. 2007:370(9590):859-77.

19. Sareen J, Jacobi F, Cox B, Belik SL, Clara I, Stein MB. Disability and poor quality of life associated with comorbid anxiety disorders and physical conditions. Arch Intern Med. 2006;166(19):2109-16.
20. Feinstein AR. The pre-therapeutic classification of co-morbidity in chronic disease. J Chronic Dis. 1970;23(7):455-68.

21. Fortin M, Bravo G, Hudon C, Vanasse A, Lapointe L. Prevalence of multimorbidity among adults seen in family practice. Ann Fam Med. 2005;3(3):223-8.

22. Barnett K, Mercer SW, Norbury M, Watt G, Wyke S, Guthrie B. Epidemiology of multimorbidity and implications for health care, research, and medical education: a cross-sectional study. Lancet. 2012;380(9836):37-43.

23. Hobbs FR, Baker M, Davies DSC. Morbidity matters: challenges for research; 2015

24. Eaton S, Collins A, Coulter A, Elwyn G, Grazin N, Roberts S. Putting patients first. BMJ. 2012;344(e20006):1-2

25. Naylor C, Parsonage M, McDaid D, Knapp M, Fossey M, Galea A. Long-term conditions and mental health: the cost of co-morbidities. London: The King's Fund; 2012.

26. Prince M, Patel V, Saxena S, Maj M, Maselko J, Phillips MR, et al. No health without mental health. Lancet. 2007;370(9590):859-77.

27. Janssen F. Cohort patterns in mortality trends among the elderly in seven European countries, 1950-99. Int J Epidemiol. 2005;34(5):1149-59.

28. Kinsella KG, Phillips DR. Global aging: the challenge of success, vol. Vol. 60 no. 1. Washington DC: Population Reference Bureau; 2005. p. 3.

29. Salisbury C, Johnson L, Purdy S, Valderas JM, Montgomery AA. Epidemiology and impact of multimorbidity in primary care: a retrospective cohort study. Br J Gen Pract. 2011;61(582):e12-21.

30. Ward BW, Schiller JS, Goodman RA. Multiple chronic conditions among US adults. Prev Chronic Dis. 2012;11:1-4.

31. Eaton $S$, Roberts $S$, Turner B. Delivering person centred care in long term conditions. BMJ. 2015;350:h181.

32. Wagner EH, Austin BT, Von Korff M. Organizing care for patients with chronic illness. Milbank Q. 1996;74(4):511-44.

33. World Health Organization. ROADMAP. Strengthening people-centred health systems in the WHO European region: a framework for action towards coordinate. World Health Organization. 2013.

34. Barr VJ, Robinson S, Marin-Link B, Underhill L, Dotts A, Ravensdale D, Salivaras S. The expanded chronic care model: an integration of concepts and strategies from population health promotion and the chronic care model. Healthc Q. 2003;7(1):73-82.

35. The Health Foundation. Person-centred care made simple. What everyone should know about person-centred care. The Health Foundation. 2014. https://www.health.org.uk/publications/person-centred-care-made-simple. Accessed 01 Oct 2017.

36. Glasby J, Dickinson H. Partnership working in health and social care: what is integrated care and how can we deliver it? London: Policy Press (UK); 2014.

37. House M. State of the nations report: terminal illness care in England, Northern Ireland, Scotland and Wales. Centre for health and social care research; 2016.

38. Department of Health. Supporting people with long term conditions to self care. A guide to developing local strategies and good practice. Department of health. 2006. https://webarchive.nationalarchives.gov.uk/20090217000112/ http://www.dh.gov.uk/en/Publicationsandstatistics/Publications/ PublicationsPolicyAndGuidance/DH_4130725. Accessed 01 Oct 2017.

39. Glasgow RE. Perceived barriers to self-management and preventative behaviors. In: Behavioral research programme. Maryland: National Cancer Institute; 2008. https://cancercontrol.cancer.gov/brp/research/constructs/ barriers.html. Accessed 01 Oct 2017

40. Chan WL, Hui E, Chan C, Cheung D, Wong S, Wong R, et al. Evaluation of chronic disease self-management programme (CDSMP) for older adults in Hong Kong. J Nutr, Health Aging. 2011;15(3):208-14.

41. Collins B. Intentional whole health system redesign. Southcentral Foundation's 'Nuka' system of care. London: The King's Fund; 2015.

42. Gottlieb K. The Nuka system of care: improving health through ownership and relationships. Int J Circumpolar Health. 2013;72(1):21118.

43. Hamar B, Wells A, Gandy W, Haaf A, Coberley C, Pope JE, et al. The impact of a proactive chronic care management program on hospital admission rates in a German health insurance society. Popul Health Manag. 2010;13(6):339-45.

44. Kreitzer MJ, Monsen KA, Nandram S, De Blok J. Buurtzorg Nederland: a global model of social innovation, change and whole-system healing. Glob Adv Health Med. 2015;4(1):40-4.

45. Morisky DE, Kominski GF, Afifi AA, Kotlerman JB. The effects of a disease management program on self-reported health behaviors and health outcomes: evidence from the "Florida: a healthy state (FAHS)" medicaid programme. Health Educ Behav. 2009;36(3):505-17. 
46. Newman S, Steed L, Mulligan K. Self-management interventions for chronic illness. Lancet. 2004;364(9444):1523-37.

47. Stock S, Drabik A, Büscher G, Graf C, Ullrich W, Gerber A, et al. German diabetes management programs improve quality of care and curb costs. Health Aff. 2010;29(12):2197-205.

48. Doherty Y, Eaton S, Turnbull R, Oliver L, Roberts S, Ludbrook S, et al. Year of care: the key drivers and theoretical basis for a new approach in diabetes care. Pract Diabetes. 2012;29(5):183

49. Bodenheimer T, Wagner EH, Grumbach K. Improving primary care for patients with chronic illness: the chronic care model, part 2. Jama. 2002; 288(15):1909-14.

50. Aliotta SL, Grieve K, Giddens JF, Dunbar L, Groves C, Frey K, et al. Guided care: a new frontier for adults with chronic conditions. Prof Case Manag. 2008;13(3):151-8.

51. Coulter A, Collins A. Making shared decision-making a reality: no decision about me, without me. London: The King's Fund; 2011

52. Hibbard J, Gilburt H. Supporting people to manage their health. An introduction to patient activation. London: The King's Fund; 2014.

53. Mulley AG, Trimble C, Elwyn G. Patients' preferences matter: stop the silent misdiagnosis. London: The King's Fund; 2012.

54. Nunes V, Neilson J, O'Flynn N, Calvert N, Kuntze S, Smithson H, et al. Medicines adherence: involving patients in decisions about prescribed medicines and supporting adherence. London: Royal College of General Practitioners; 2009.

55. O'Connor AM, Llewellyn-Thomas HA, Flood AB. Modifying unwarranted variations in health care: shared decision making using patient decision aids. Health Aff. 2004;23(2);VAR63-72.

56. Rathert C, Wyrwich MD, Boren SA. Patient-centered care and outcomes: a systematic review of the literature. Med Care Res Rev. 2013;70(4):351-79.

57. The Health Foundation. Helping people help themselves. A review of the evidence considering whether it is worthwhile to support self-management. The Health Foundation. 2011. http://www.health.org.uk/sites/health/files/ HelpingPeopleHelpThemselves.pdf.. Accessed 01 Oct 2017.

58. The Health Foundation. Helping people share decision making. A review of evidence considering whether shared decision making is worthwhile. The Health Foundation. 2012. https://www.health.org.uk/sites/default/files/ HelpingPeopleShareDecisionMaking.pdf. Accessed 01 Oct 2017.

59. The King's Fund. How to deliver high-quality, patient-centred, cost-effective care. Consensus solutions from the voluntary sector. London: The King's Fund; 2010.

60. Kennedy A, Rogers A, Bowen R, Lee V, Blakeman T, Gardner C, et al. Implementing, embedding and integrating self-management support tools for people with long-term conditions in primary care nursing: a qualitative study. Int J Nurs Stud. 2014;51(8):1103-13.

61. Goodwin N, Sonola L, Thiel V, Kodner D. Co-ordinated care for people with complex chronic conditions. Key lessons and markers for success. London: The King's Fund; 2013.

62. Audulv $\AA$, Norbergh KG, Asplund K, Hörnsten $\AA$. An ongoing process of inner negotiation-a grounded theory study of self-management among people living with chronic illness. J Nurs Healthc Chronic IIIn. 2009;1(4): 283-93.

63. Funnell MM, Anderson RM. Empowerment and self-management of diabetes. Clin Diab. 2004:22(3):123-7.

64. Batalden M, Batalden P, Margolis P, Seid M, Armstrong G, Opipari-Arrigan L, et al. Coproduction of healthcare service. BMJ Qual Saf. 2016;25:509-17.

65. Batalden PB, Davidoff F. What is "quality improvement" and how can it transform healthcare. Healthcare. 2007;16(1):2-3.

66. Blakeman $T$, Bower $P$, Reeves $D$, Chew-Graham C. Bringing self-management into clinical view: a qualitative study of long-term condition management in primary care consultations. Chronic Illness. 2010;6(2):136-50.

67. Blakeman T, Macdonald W, Bower P, Gately C, Chew-Graham C. A qualitative study of GPs' attitudes to self-management of chronic disease. $\mathrm{Br}$ J Gen Pract. 2006;56(527):407-14.

68. Davies NJ. Improving self-management for patients with long-term conditions. Nurs Stand. 2010;24(25):48-56.

69. Kennedy A, Bower P, Reeves D, Blakeman T, Bowen R, Chew-Graham C, et al. Implementation of self management support for long term conditions in routine primary care settings: cluster randomised controlled trial. BMJ. 2013; 346:f2882

70. Kennedy A, Rogers A, Bower P. Support for self-care for patients with chronic disease. BMJ. 2007;335(7627):968.
71. MacDonald W, Rogers A, Blakeman T, Bower P. Practice nurses and the facilitation of self-management in primary care. J Adv Nurs. 2008;62(2):191-9.

72. West E, Barron DN, Reeves R. Overcoming the barriers to patient-centred care: time, tools and training. J Clin Nurs. 2005;14(4):435-43.

73. Bonomi AE, Wagner EH, Glasgow RE, VonKorff M. Assessment of chronic illness care $(\mathrm{ACIC})$ : a practical tool to measure quality improvement. Health Serv Res. 2002;37(3):791-830

74. Glasgow RE, Wagner EH, Schaefer J, Mahoney LD, Reid RJ, Greene SM. Development and validation of the patient assessment of chronic illness care (PACIC). Med Care. 2005;43(5);436-44.

75. Hudon C, Fortin M, Haggerty JL, Lambert M, Poitras ME. Measuring patients' perceptions of patient-centred care: a systematic review of tools for family medicine. Ann Fam Med. 2011;9(2):155-64.

76. Ogden J. Health psychology. London: McGraw-Hill Education (UK); 2012

77. Ajzen I. The theory of planned behaviour. Organ Behav Hum Decis Process. 1991;50(2):179-211.

78. Ajzen I. Perceived behavioural control, self-efficacy, locus of control, and the theory of planned behavior. J Appl Soc Psychol. 2002;32(4):665-83.

79. Sniehotta FF, Presseau J, Araújo-Soares V. Time to retire the theory of planned behaviour. Health Psychol Rev. 2014;8(1):1-7.

80. Eccles MP, Hrisos S, Francis J, Kaner EF, Dickinson HO, Beyer F, et al. Do selfreported intentions predict clinicians' behaviour: a systematic review. Implement Sci. 2006;1(1):28.

81. Godin G, Bélanger-Gravel A, Eccles M, Grimshaw J. Healthcare professionals' intentions and behaviours: a systematic review of studies based on social cognitive theories. Implement Sci. 2008;3(1):36.

82. Perkins MB, Jensen PS, Jaccard J, Gollwitzer P, Oettingen G, Pappadopulos E, et al. Applying theory-driven approaches to understanding and modifying clinicians' behavior: what do we know? Psychiatr Serv. 2007;58(3):342-8.

83. Russo DA, Stochl J, Croudace TJ, Graffy JP, Youens J, Jones PB, et al. Use of the theory of planned behaviour to assess factors influencing the identification of individuals at ultra-high risk for psychosis in primary care. Early Interv Psychiatry. 2012;6(3):265-75

84. Wakefield JG, McLaws ML, Whitby M, Patton L. Patient safety culture: factors that influence clinician involvement in patient safety behaviours. Qual Saf Health Care. 2010:19(6):585-91.

85. White KM, Jimmieson NL, Obst L, Graves N, Barnett A, Cockshaw W, et al. Using a theory of planned behaviour framework to explore hand hygiene beliefs at the ' 5 critical moments' among Australian hospital-based nurses. BMC Health Serv Res. 2015;15(1):59.

86. Francis J, Eccles MP, Johnston M, Walker AE, Grimshaw JM, Foy R, et al. Constructing questionnaires based on the theory of planned behaviour: a manual for health services researchers. Newcastle upon Tyne: Centre for Health Services Research, University of Newcastle upon type (UK); 2004.

87. Kitzinger J. Qualitative research. Introducing focus groups. BMJ. 1995; 7000:299.

88. Kitzinger J. Using focus groups to understand experiences of health and illness. In: Understanding and using health experiences: improving patient care, vol. 1; 2013. p. 59.

89. Kreuger RA, Casey MA. Designing and conducting focus group interviews. Soc Anal Select Tools Techn. 2002;4(23):4-24.

90. Rabiee F. Focus-group interview and data analysis. Proc Nutr Soc. 2004; 63(4):65-0.

91. Boyatzis RE. Transforming qualitative information: thematic analysis and code development. London: Sage (UK); 1998.

92. Braun V, Clarke V. Using thematic analysis in psychology. Qual Res Psychol. 2006:3(2):77-101.

93. Bono C, Ried LD, Kimberlin C, Vogel B. Missing data on the center for epidemiological studies depression scale: a comparison of 4 imputation techniques. Res Soc Adm Pharm. 2007;3(1):1-27.

94. Downey RG, King CV. Missing data in likert ratings: a comparison of replacement methods. J Gen Psychol. 1998;125(2):175-91.

95. Fairclough DL, Cella DF. Functional assessment of cancer therapy (FACT-G): non-response to individual questions. Qual Life Res. 1996;5(3):321-9.

96. Roth PL, Switzer FS III, Switzer DM. Missing data in multiple item scales: a Monte Carlo analysis of missing data techniques. Organ Res Methods. 1999; 2(3):211-32

97. Schafer JL. Multiple imputation: a primer. Stat Methods Med Res. 1999;8(1):3-15.

98. Shrive FM, Stuart H, Quan H, Ghali WA. Dealing with missing data in a multi-question depression scale: a comparison of imputation methods. BMC Med Res Methodol. 2006;6(1):57. 
99. Montano DE, Kasprzyk D. Theory of reasoned action, theory of planned behaviour, and the integrated behavioral model. In: Health behaviour: theory, research and practice; 2015. p. 95-124.

100. Ajzen I. The theory of planned behaviour is alive and well, and not ready to retire: a commentary on Sniehotta, Presseau, and Araújo-Soares. Health Psychol Rev. 2015;9(2):131-7.

101. Bergman MM. Advances in mixed methods research: theories and applications. 1st ed. London: Sage (UK); 2008.

102. Johnson RB, Onwuegbuzie AJ, Turner LA. Towards a definition of mixed methods research. J Mixed Methods Res. 2007;1 (2):112-33.

103. Sale JE, Lohfeld LH, Brazil K. Revisiting the quantitative-qualitative debate: implications for mixed-methods research. Qual Quant. 2002;36(1):43-54.

104. Skills for health. Urban and rural issues for the health sector in Scotland. Skills for health. 2010. http://www.skillsforhealth.org.uk/index.php?option= com_mtree\&task=att_download\&link_id=98\&cf_id=24. Accessed $06 \mathrm{Mar}$ 2019

\section{Publisher's Note}

Springer Nature remains neutral with regard to jurisdictional claims in published maps and institutional affiliations.

Ready to submit your research? Choose BMC and benefit from:

- fast, convenient online submission

- thorough peer review by experienced researchers in your field

- rapid publication on acceptance

- support for research data, including large and complex data types

- gold Open Access which fosters wider collaboration and increased citations

- maximum visibility for your research: over $100 \mathrm{M}$ website views per year

At BMC, research is always in progress.

Learn more biomedcentral.com/submissions 